\title{
3 Research Suare

\section{Influence of Oral Health on Frailty in Patients With Type 2 Diabetes Aged 75 Years or Older}

Masaki Ishii ( $\square$ ishii-tky@umin.ac.jp )

The University of Tokyo

\section{Yasuhiro Yamaguchi}

Jichi Medical University Saitama Medical Center

Hironobu Hamaya

The University of Tokyo

\section{Yuko Iwata}

The University of Tokyo

Kazufumi Takada

The University of Tokyo

\section{Sumito Ogawa}

The University of Tokyo

Mitsuo Imura

Okamoto Internal Medicine Clinic

Masahiro Akishita

The University of Tokyo

\section{Research Article}

Keywords: elderly, frailty, type 2 diabetes, oral health, oral frailty index-8

Posted Date: March 22nd, 2021

DOI: https://doi.org/10.21203/rs.3.rs-330526/v1

License: (c) (i) This work is licensed under a Creative Commons Attribution 4.0 International License.

Read Full License 


\section{Abstract}

Background: The purpose of this study was to evaluate the influence of oral health on frailty in elderly patients with type 2 diabetes.

Methods: Patients with type 2 diabetes aged 75 years or older were included in this retrospective study. Eligible patients were surveyed by questionnaire for frailty, oral health status, and cognitive and living functions. Factors influencing pre-frailty, frailty, and individual frailty score categories were evaluated.

Results: Of the 111 patients analyzed, 66 cases (59.5\%) were categorized as robust, 33 cases $(29.7 \%)$ as pre-frailty, and 5 cases $(4.5 \%)$ as frailty. The oral frailty index, the cognitive and living functions score, and BMI were factors influencing pre-frailty or frailty. In the evaluation by individual frailty score categories, BMI was found to only influence those with a frailty score $\leq 2$. The cognitive and living functions score was a factor influencing those with frailty scores $\leq 3$. The oral frailty index was found to have a significant influence on all frailty score categories.

Conclusion: Poor oral health has an influence on frailty in patients with type 2 diabetes aged $\geq 75$. The management of oral health is an important consideration in the prevention of the frailty of elderly patients with diabetes.

\section{Introduction}

Frailty is a condition in which physical and mental vulnerability develops with aging and has a great impact on the prognosis of the elderly. There are various risk factors for frailty, such as underlying disease, nutritional status, and sarcopenia, which interact with each other to develop vulnerability ${ }^{1}$. Poor oral health conditions are known to affect frailty in the elderly. Kamden et al. reported that decreased masticatory function was a significant influential factor for frailty ${ }^{2}$. Tanaka et al. reported that poor oral health status evaluated based on masticatory function, tongue pressure, salivation, and so on, was a significant risk factor for the later onset of frailty ${ }^{3}$. It has been suggested that the influence of poor oral health on frailty involves tooth loss, decreased salivation, periodontal disease, and dental caries ${ }^{4}$. Loss of teeth reduces masticatory function and worsens nutritional status 5,6 . Saliva secretion is reduced by aging, medication such as anti-hypertensives and anti-depressants, or radiation therapy ${ }^{4}$, and the decrease in saliva is likely to cause dental caries, leading to decreased chewing and swallowing functions. Poor oral health including periodontal disease and dental caries reduces masticatory function and worsens nutritional status through tooth loss ${ }^{7}$.

Poor glycemic control in patients with diabetes has been reported to exacerbate various oral health conditions including dental caries, decreased salivation and periodontal disease ${ }^{8}$. Izuora et al. reported that in diabetic patients averaged 58.9 years old, only $6.4 \%$ had their original 32 teeth and $15.3 \%$ had lost all of their teeth ${ }^{9}$. As periodontal disease progresses, it leads to tooth loss, and the prevalence of periodontal disease is higher in diabetics than in the general population ${ }^{10}$. Periodontal disease also 
causes systemic inflammation and adversely affects diabetes through insulin resistance ${ }^{11}$. In addition, diabetic patients reportedly have decreased salivation, $46 \%$ of which had dry mouth ${ }^{12}$. As described above, these various changes in the oral cavity of diabetic patients interact with each other, resulting in deterioration of masticatory and swallowing function.

Diabetes itself is reportedly to be a risk factor for frailty. Insulin is involved in promoting protein synthesis and suppressing proteolysis but, with diabetes, insulin resistance suppresses protein synthesis, affecting muscle strength and motor function ${ }^{13,14}$. Diabetes is a risk factor for both poor oral health and frailty, and, therefore, oral health status may affect frailty in diabetic patients more than in the general population.

The impact of nutritional status on health may vary with age of patient. In Japan, the elderly aged 75 and over are termed the 'late-stage elderly' in the medical system. In a study of diabetic patients in Japan, Yamaoka et al. found that reduced protein intake was a significant risk factor for all-cause mortality only in patients aged 75 years and older, and thus adequate nutrition is required especially for patients aged 75 and older ${ }^{15}$. A strict diet to prevent obesity is important for younger patients with diabetes, but prevention of frailty and sarcopenia becomes more important as they age ${ }^{16}$. Against this background, we evaluated the effect of oral health on frailty in patients with type 2 diabetes aged 75 years and older.

\section{Results}

\section{Patient characteristics}

Of the patients treated for type 2 diabetes from December 2019 to March 2020, 229 patients aged 75 years and older were enrolled; 81 were excluded because of gait disturbance (Fig. 1). Questionnaire surveys on the oral frailty index-8 (OFI-8), frailty scoring, and the Dementia Assessment Sheet for Community-based Integrated Care System-8 items (DASC-8) were conducted in the 148 patients without gait disturbance. Of those, 37 patients were excluded due to incomplete answers, and the remaining 111 patients were analyzed. The mean age was 79.7 years, with an average duration of diabetes of 20.4 years, of which 54 (48.6\%) had a duration of diabetes of 20 years or more (Table 1). Regarding frailty, 66 $(59.5 \%)$ cases were defined as robust, $33(29.7 \%)$ cases as pre-frailty, and $5(4.5 \%)$ as frailty. The rate for each category of the DASC-8 was $72.1 \%$ (I), $22.5 \%$ (II) and $5.4 \%$ (III). Significant differences were found in sex, age, OFI-8 score, and DASC-8 category in multiple comparisons among the robust, pre-frailty, and frailty groups.

\section{Factors influencing frailty}

Multiple logistic analysis showed that OFI-8 score, DASC-8 score, and BMI were factors influencing prefrailty, and that OFI-8 score and DASC-8 were factors influencing frailty (Table 2). The adjusted odds with $95 \%$ confidence interval $(95 \% \mathrm{Cl})$ of the OFI-8 scores were $1.34[1.04,1.72]$ for pre-frailty and $1.55[1.10$, 2.18] for frailty (Fig. 2a). In the evaluation by individual frailty score categories, BMI was not found to be 
a factor influencing a frailty scores $\geq 3$ (Fig. 2b). The DASC-8 score was not a factor influencing a frailty scores $\geq 4$. The OFI-8 score was a significant factor influencing all frailty score categories. The adjusted odds ratios with $95 \% \mathrm{Cl}$ of OFI-8 scores were $1.34[1.04,1.72]$ for frailty scores $\geq 1,1.45[1.05,2.00]$ for scores $\geq 2,1.55[1.10,2.18]$ for scores $\geq 3$ and $7.09[1.08,46.6]$ for scores $\geq 4$.

Factors influencing the OFI-8 score

Multiple regression analysis showed that frailty scores and duration of diabetes were significant influencing factors on the OFI-8 score (Table 2). The cut-off value of the duration of diabetes for oral frailty from ROC analysis was 15 years (AUC: 0.62 ). The proportion of patients with oral frailty was $38 \%$ in duration of diabetes $\leq 15$ years and $62 \%$ in that of $>15$ years (Fig. 3a). The proportion of patients with oral frailty by sex and duration of diabetes were $22 \%$ ( $\leq 15$ years) vs. $57 \%$ (> 15 years) in male and $59 \%$ vs. $73 \%$ in female (Figs. $3 \mathrm{~b}$ and $3 \mathrm{c}$ ).

\section{Discussion}

The influence of oral health on frailty was investigated in 111 patients with type 2 diabetes aged 75 years and older. A higher OFI-8 score was a significant factor influencing all frailty score categories.

In the elderly, poor oral health represented by deterioration of masticatory function and swallowing function has been shown to affect sarcopenia and frailty 4,17 . Elderly people with impaired masticatory function often avoid eating hard-to-eat foods such as meat, fruits, and vegetables ${ }^{18-20}$, and these changes in eating habits increase the risk of malnutrition and frailty ${ }^{17,21}$. Our results support the results of these previous studies. To our knowledge, this is the first report showing the relationship between oral health and frailty in diabetic patients aged $\geq 75$.

In this study, the OFI-8 score, DASC-8 score and BMI were shown to be factors influencing pre-frailty or frailty. High BMI has been reported to be a risk factor for frailty ${ }^{22-24}$. Excessive lipid deposition and infiltration of lipids into muscle fibers affect muscle weakness and activity ${ }^{25}$, and excessive lipid deposition has been suggested to increase the risk of frailty through systemic inflammation ${ }^{26}$. In our study, BMI was found to be a risk factor for pre-frailty, suggesting that prevention of obesity and metabolic syndrome is important for the elderly or middle-aged parson even for those in the robust status prior to pre-frailty.

On the other hand, our study showed that BMI was not a significant risk factor for frailty or frailty scores $\geq 3$. As mentioned above, previous studies have shown that BMI was a risk factor for frailty $22-24$, but the studies by Niederstrasser et al. and Hubbard et al. were conducted in subjects aged $\geq 50^{22,24}$, and the study by Watanabe et al. was conducted in subjects aged $\geq 65^{23}$. Our study was conducted in patients with diabetes aged $\geq 75$ and is a population at higher risk of frailty than those in previous studies. Considering the above, the influence of BMI on frailty may vary depending on patient age and severity of frailty. In the evaluation by individual frailty score categories, BMI was not a factor influencing frailty 
scores $\geq 3$ and DASC-8 was not a factor influencing frailty scores $\geq 4$, but the OFI-8 score was consistently a significant factor influencing all categories of frailty scores. These results indicate that as frailty progresses, the impact of oral health on the frailty increases and the effects of BMI and DASC-8 relatively decrease. There is an interaction between diabetes and oral health ${ }^{8,10}$. Therefore, the impact of oral health on frailty in patients with diabetics may be relatively higher than in the general population. The fact that duration of diabetes had a significant influence on the OFI-8 score in this study supports this speculation.

In our study, the cut-off value for the duration of diabetes for oral frailty defined as OFI-8 score $\geq 4{ }^{27}$ was 15 years. The proportion of patients with oral frailty by sex and duration of diabetes were $22 \%(\leq 15$ years) vs. $57 \%$ (>15 years) in the male and $59 \%$ vs. $73 \%$ in the female, indicating that the female patients develop oral frailty earlier than the male. The tongue pressure, one of the indicators of oral function, may have contribute to this result. The tongue is mostly composed of muscle and an association between arm muscle mass and tongue thickness has been shown ${ }^{28}$. It has also been reported that tongue pressure and tongue thickness are significantly lower in women compared to men ${ }^{29,30}$. Considering these facts, the function of the tongue relative to muscle mass may be one of the causes of the differences between the sexes in the development of oral frailty.

There are several limitations to this study. This was a retrospective study conducted in a single institution. The OFI-8 score as an index of oral health is based on a questionnaire, and oral function including masticatory function and swallowing function was not evaluated by dental or oral specialists. The results of this study need to be interpreted with these factors in mind.

In conclusion, this is the first study to show that oral health influences frailty in diabetic patients aged 75 years and older. Oral conditions are affected by the duration of diabetes and, therefore, it is important to manage oral health for the prevention of frailty in elderly patients with diabetes.

\section{Methods}

Patients

This is a retrospective study of data from Okamoto Internal Medicine Clinic, Shizuoka, Japan, collected from December 2019 to March 2020. The subjects of this study were patients with type 2 diabetes aged 75 years and older, and excluded patients with gait disturbance.

This study was conducted in compliance with the Declaration of Helsinki and according to the Ethical Guidelines for Medical and Health Research Involving Human Subjects established by the Ministry of Health, Labour, and Welfare in Japan. The study protocol was approved by the ethical review committee of the Seishinkai Group and Okamoto Internal Medicine Clinic. Written informed consent was obtained from all study patients.

Methods 
Of the 229 patients enrolled, 111 eligible patients were surveyed by questionnaire for frailty, oral health status, and cognitive and living functions (Fig. 1). OFI-8, an eight-question oral health assessment tool reported by Tanaka et al., was used ${ }^{27}$. The adjusted odds ratio with a $95 \% \mathrm{Cl}$ of the OFI-8 score to frailty was estimated. Adjusted odds ratios were calculated for pre-frailty, frailty, and individual frailty score categories. In addition, factors influencing oral health were examined. Data on age, sex, BMI, duration of diabetes, and $\mathrm{HbA} 1 \mathrm{c}$ were collected.

For the evaluation of frailty, a self-evaluation questionnaire developed by Yamada et al. consisting of five items -weight loss, gait speed, exercise frequency, short-term memory, and feeling of fatigue-was used 31. Cases corresponding to 1 or 2 items were defined as pre-frailty, and cases corresponding to 3 or more items were defined as frailty.

Cognitive and living functions were evaluated using the DASC-8 developed by Toyoshima et al ${ }^{32}$. Patients were classified into three categories (I, II, and III) determined by DASC-8 score-I: normal cognitive function and ADL independence; II: mild cognitive impairment or mild dementia or instrumental ADL decline, and basic ADL independence; and III: moderate or severe dementia or decreased basic ADL or many comorbidities or dysfunctions ${ }^{32}$.

The OFI-8 score was the sum of the scores for each of the following questions below. Q1: Do you have any difficulties eating tough foods compared to 6 months ago? (2 points for Yes), Q2: Have you choked on your tea or soup recently? (2 points for Yes), Q3: Do you use dentures? (2 points for Yes), Q4: Do you often have a dry mouth? (1 point for Yes), Q5: Do you go out less frequently than you did last year? (1 point for Yes), Q6: Can you eat hard foods like squid jerky or pickled radish? (1 point for No), Q7: How many times do you brush your teeth in a day? (3 or more times/day) (1 point for No), and Q8: Do you visit a dental clinic at least annually? (1 point for No) ${ }^{27}$.

Statistics

Descriptive statistics are expressed as $n(\%)$ and mean \pm SD. The Kruskal-Wallis test was used to compare continuous variables, and the chi-square test was used to compare categorical variables. For the evaluation of factors influencing pre-frailty, frailty and individual frailty score categories, the adjusted odds ratio $[95 \% \mathrm{Cl}]$ was calculated from multiple logistic analysis using the variables extracted by the stepwise method from the following variables: sex, age, duration of diabetes, HbA1c, OFI-8, DASC-8, and BMI. For the evaluation of the factors influencing the OFI-8 scores, multiple regression analysis was used using the variables extracted by the stepwise method from the following variables: sex, age, BMI, duration of diabetes, HbA1c, DASC-8, and frailty score. For the influence of diabetic duration on oral health, an OFI8 score $\geq 4$ was defined as oral frailty ${ }^{27}$ and a cutoff value was calculated using ROC analysis. The twosided a was set to be 0.05 . Statistical analysis was performed using SPSS (IBM).

\section{Declarations}




\section{Author Contributions}

M.I., Y.Y., H.H., Y.I. and K.T. conceived and designed the research, reviewed data; S.O. and M.A. made critical revision of the manuscript; M.I. and M.I. collected data, performed the statistical analysis and drafted the manuscript. All authors had reviewed the manuscript.

\section{Conflict of Interest}

The authors have declared no conflicts of interest associated with this article.

\section{Sponsor's Role}

We have no sponsors to disclose.

Data availability

The datasets analyzed will be available from the corresponding author on reasonable request.

\section{References}

1. Ng, T.P., Feng, L., Nyunt, M.S., Larbi, A., Yap, K.B., Frailty in older persons: multisystem risk factors and the Frailty Risk Index (FRI). J Am Med Dir Assoc. 15, 635-42 (2014).

2. Kamdem, B., Seematter-Bagnoud, L., Botrugno, F., Santos-Eggimann, B. Relationship between oral health and Fried's frailty criteria in community-dwelling older persons. BMC Geriatr. 17, 174 (2017).

3. Tanaka, T. et al. Oral Frailty as a Risk Factor for Physical Frailty and Mortality in Community-Dwelling Elderly. J Gerontol A Biol Sci Med Sci. 73, 1661-1667 (2018).

4. Azzolino, D. et al. Poor Oral Health as a Determinant of Malnutrition and Sarcopenia. Nutrients. 11, 2898 (2019).

5. Musacchio, E. et al. Tooth loss in the elderly and its association with nutritional status, socioeconomic and lifestyle factors. Acta Odontol Scand. 65, 78-86 (2007).

6. Felton, D.A. Complete Edentulism and Comorbid Diseases: An Update. J Prosthodont. 25, 5-20 (2016).

7. Azzolino, D. et al. Poor Oral Health as a Determinant of Malnutrition and Sarcopenia. Nutrients. 11, 2898 (2019).

8. Mauri-Obradors, E., Estrugo-Devesa, A., Jané-Salas, E., Viñas, M., López-López, J. Oral manifestations of Diabetes Mellitus. A systematic review. Med Oral Patol Oral Cir Bucal. 22, e586-e594 (2017).

9. Izuora, K.E. et al. DENTAL LOSS AMONG AMBULATORY PATIENTS WITH DIABETES. J Clin Trans/ Endocrinol. 4, 28-31 (2016). 
10. Wu, C. Z. et al. Epidemiologic relationship between periodontitis and type 2 diabetes mellitus. $B M C$ Oral Health. 20, 204 (2020).

11. Indurkar, M. S., Maurya, A. S., Indurkar, S. Oral Manifestations of Diabetes. Clin Diabetes. 34, 54-7 (2016).

12. Lessa, L. S. et al. Meta-Analysis of Prevalence of Xerostomia in Diabetes Mellitus. Int. Arch. Med. 8, 1-13 (2015).

13. Rolland, Y. et al. Sarcopenia: its assessment, etiology, pathogenesis, consequences and future perspectives. J Nutr Health Aging. 12, 433-50 (2008).

14. Yanase, T., Yanagita, I., Muta, K., Nawata, H. Frailty in elderly diabetes patients. Endocr J. 65, 1-11 (2018).

15. Yamaoka, T. et al. Association between Low Protein Intake and Mortality in Patients with Type 2 Diabetes. Nutrients. 12, 1629 (2020).

16. Tamura, Y., Omura, T., Toyoshima, K., Araki, A. Nutrition Management in Older Adults with Diabetes: A Review on the Importance of Shifting Prevention Strategies from Metabolic Syndrome to Frailty. Nutrients. 12, 3367 (2020).

17. Takahashi, M., Maeda, K., Wakabayashi, H. Prevalence of sarcopenia and association with oral health-related quality of life and oral health status in older dental clinic outpatients. Geriatr Gerontol Int. 18, 915-921 (2018).

18. Gil-Montoya, J. A., de Mello, A. L., Barrios, R., Gonzalez-Moles, M. A., Bravo, M. Oral health in the elderly patient and its impact on general well-being: a nonsystematic review. Clin Interv Aging. 10, 461-7 (2015).

19. Hutton, B., Feine, J., Morais, J. Is there an association between edentulism and nutritional state? $J$ Can Dent Assoc. 68, 182-7 (2002).

20. Hung, H. C., Colditz, G., Joshipura, K. J. The association between tooth loss and the self-reported intake of selected CVD-related nutrients and foods among US women. Community Dent Oral Epidemiol 2005;33:167-73.

21. Woo, J., Tong, C., Yu, R. Chewing Difficulty Should be Included as a Geriatric Syndrome. Nutrients. 10, 1997 (2018).

22. Niederstrasser, N. G., Rogers, N. T., Bandelow, S. Determinants of frailty development and progression using a multidimensional frailty index: Evidence from the English Longitudinal Study of Ageing. PLoS One. 14, e0223799 (2019).

23. Watanabe, D. et al. A U-Shaped Relationship Between the Prevalence of Frailty and Body Mass Index in Community-Dwelling Japanese Older Adults: The Kyoto-Kameoka Study. J Clin Med. 9, 1367 (2020).

24. Hubbard, R. E., Lang, I. A., Llewellyn, D. J., Rockwood, K. Frailty, body mass index, and abdominal obesity in older people. J Gerontol A Biol Sci Med Sci. 65, 377-81 (2010). 
25. Porter, Starr, K. N., McDonald, S. R., Bales, C. W. Obesity and physical frailty in older adults: a scoping review of lifestyle intervention trials. J Am Med Dir Assoc. 15, 240-50 (2014).

26. Shoelson, S. E., Herrero, L., Naaz, A. Obesity, inflammation, and insulin resistance. Gastroenterology. $132,2169-80$ (2007).

27. Tanaka, T., Hirano, H., Ohara, Y., Nishimoto, M., lijima, K. Oral Frailty Index-8 in the risk assessment of new-onset oral frailty and functional disability among community-dwelling older adults. https://www.sciencedirect.com/science/article/abs/pii/S0167494321000030 (in press).

28. Tamura, F., Kikutani, T., Tohara, T., Yoshida, M., Yaegaki, K. Tongue thickness relates to nutritional status in the elderly. Dysphagia. 27, 556-61 (2012).

29. Nagayoshi, M. et al. Social networks, leisure activities and maximum tongue pressure: crosssectional associations in the Nagasaki Islands Study. BMJ Open. 7, e014878 (2017).

30. Nakamori, M. et al. Tongue thickness measured by ultrasonography is associated with tongue pressure in the Japanese elderly. PLoS One. 15, e0230224 (2020).

31. Yamada, M., Arai, H. Predictive Value of Frailty Scores for Healthy Life Expectancy in CommunityDwelling Older Japanese Adults. J Am Med Dir Assoc. 16, 1002.e7-11 (2015).

32. Toyoshima, K. et al. Development of the Dementia Assessment Sheet for Community-based Integrated Care System 8-items, a short version of the Dementia Assessment Sheet for Communitybased Integrated Care System 21-items, for the assessment of cognitive and daily functions. Geriatr Gerontol Int. 18, 1458-1462 (2018).

\section{Tables}

Table 怄 Patient characteristics 


\begin{tabular}{|c|c|c|c|c|c|}
\hline & $\begin{array}{l}\text { Total } \\
(n=111)\end{array}$ & $\begin{array}{l}\text { Robust } \\
(n=66)\end{array}$ & $\begin{array}{l}\text { Pre-frailty } \\
(n=33)\end{array}$ & $\begin{array}{l}\text { Frailty } \\
(n=12)\end{array}$ & $\mathrm{p}$ \\
\hline Sex (male), $\mathrm{n}(\%)$ & $72(64.9 \%)$ & 49 (74.2\%) & $18(54.5 \%)$ & $5(41.7 \%)$ & 0.032 \\
\hline Age (y.o.), mean $\pm S D$ & $79.7 \pm 3.8$ & $78.8 \pm 3.1$ & $80.7 \pm 4.4$ & $82.3 \pm 3.5$ & 0.004 \\
\hline $\mathrm{BMI}$, mean $\pm \mathrm{SD}$ & $23.2 \pm 3.8$ & $22.8 \pm 3.8$ & $23.9 \pm 4.2$ & $23.3 \pm 2.5$ & 0.336 \\
\hline $\begin{array}{l}\text { Duration of diabetes (years), } \\
\text { mean } \pm S D\end{array}$ & $20.4 \pm 11.1$ & $19.7 \pm 10.5$ & $19.5 \pm 10.6$ & $26.4 \pm 14.5$ & 0.182 \\
\hline$\leq 20$ years, $\mathrm{n}(\%)$ & $57(51.4 \%)$ & $35(53.0 \%)$ & $19(57.6 \%)$ & $3(25.0 \%)$ & \multirow[t]{2}{*}{0.131} \\
\hline$>20$ years, $\mathrm{n}(\%)$ & $54(48.6 \%)$ & $31(47.0 \%)$ & $14(42.4 \%)$ & $9(75.0 \%)$ & \\
\hline HbA1c (\%), mean \pm SD & $7.1 \pm 0.7$ & $7.1 \pm 0.6$ & $7.3 \pm 0.9$ & $7.2 \pm 0.6$ & 0.615 \\
\hline Frailty score, mean $\pm S D$ & $0.8 \pm 1.1$ & $0 \pm 0$ & $1.5 \pm 0.5$ & $3.3 \pm 0.5$ & $<0.001$ \\
\hline OFI-8 score, mean $\pm S D$ & $3.9 \pm 2.0$ & $3.3 \pm 1.8$ & $4.4 \pm 1.8$ & $5.8 \pm 2.4$ & $<0.001$ \\
\hline Score <4, n (\%) & $52(46.8 \%)$ & $39(59.1 \%)$ & $11(33.3 \%)$ & $2(16.7 \%)$ & \multirow[t]{2}{*}{0.003} \\
\hline Score $\geq 4, n(\%)$ & $59(53.2 \%)$ & $27(40.9 \%)$ & $22(66.7 \%)$ & $10(83.3 \%)$ & \\
\hline DASC-8, mean $\pm S D$ & $10.2 \pm 3.0$ & $9.2 \pm 1.3$ & $11.1 \pm 3.7$ & $13.6 \pm 4.4$ & $<0.001$ \\
\hline Category I, n (\%) & $80(72.1 \%)$ & $57(86.4 \%)$ & $19(57.6 \%)$ & $4(33.3 \%)$ & \multirow[t]{3}{*}{ - } \\
\hline Category II, n (\%) & $25(22.5 \%)$ & $9(13.6 \%)$ & $11(33.3 \%)$ & $5(41.7 \%)$ & \\
\hline Category III, n (\%) & $6(5.4 \%)$ & 0 & $3(9.1 \%)$ & 3 (25.0\%) & \\
\hline
\end{tabular}

DASC-8: the dementia assessment sheet for community-based integrated care system-8 items, OFI-8 score: oral frailty index-8

Table $\triangle \mathbb{Q}$ Factors influencing pre-frailty, frailty, and oral frailty score 


\begin{tabular}{|c|llll|}
\hline \multicolumn{2}{|c|}{ Factors influencing pre-frailty } & & & \\
\hline Variables & Estimate & S.E. & Chi-square & $p$ \\
\hline Sex (male) & -0.39 & 0.25 & 2.43 & 0.119 \\
\hline Age & 0.08 & 0.07 & 1.33 & 0.248 \\
\hline OFI-8 score & 0.28 & 0.12 & 5.04 & 0.024 \\
\hline DASC-8 score & 0.38 & 0.13 & 8.57 & 0.003 \\
\hline BMI & 0.13 & 0.06 & 4.67 & 0.030 \\
\hline Factors influencing frailty & & & & \\
\hline Variables & Estimate & S.E. & Chi-square & $\mathrm{p}$ \\
\hline OFI-8 score & 0.44 & 0.17 & 6.41 & 0.011 \\
\hline DASC-8 & 0.24 & 0.08 & 7.52 & 0.006 \\
\hline Factors influencing OFI-8 score & & & \\
\hline Variables & Estimate & S.E. & t value & $\mathrm{p}$ \\
\hline Sex (male) & -0.25 & 0.18 & -1.4 & 0.164 \\
\hline Age & 0.05 & 0.04 & 1.23 & 0.221 \\
\hline Duration of diabetes & 0.03 & 0.01 & 2.28 & 0.024 \\
\hline HbA1c & -0.36 & 0.24 & -1.52 & 0.132 \\
\hline Frailty score & 0.60 & 0.16 & 3.57 & $<0.001$ \\
\hline
\end{tabular}

DASC-8: the dementia assessment sheet for community-based integrated care system-8 items, OFI-8: oral frailty index-8

\section{Figures}




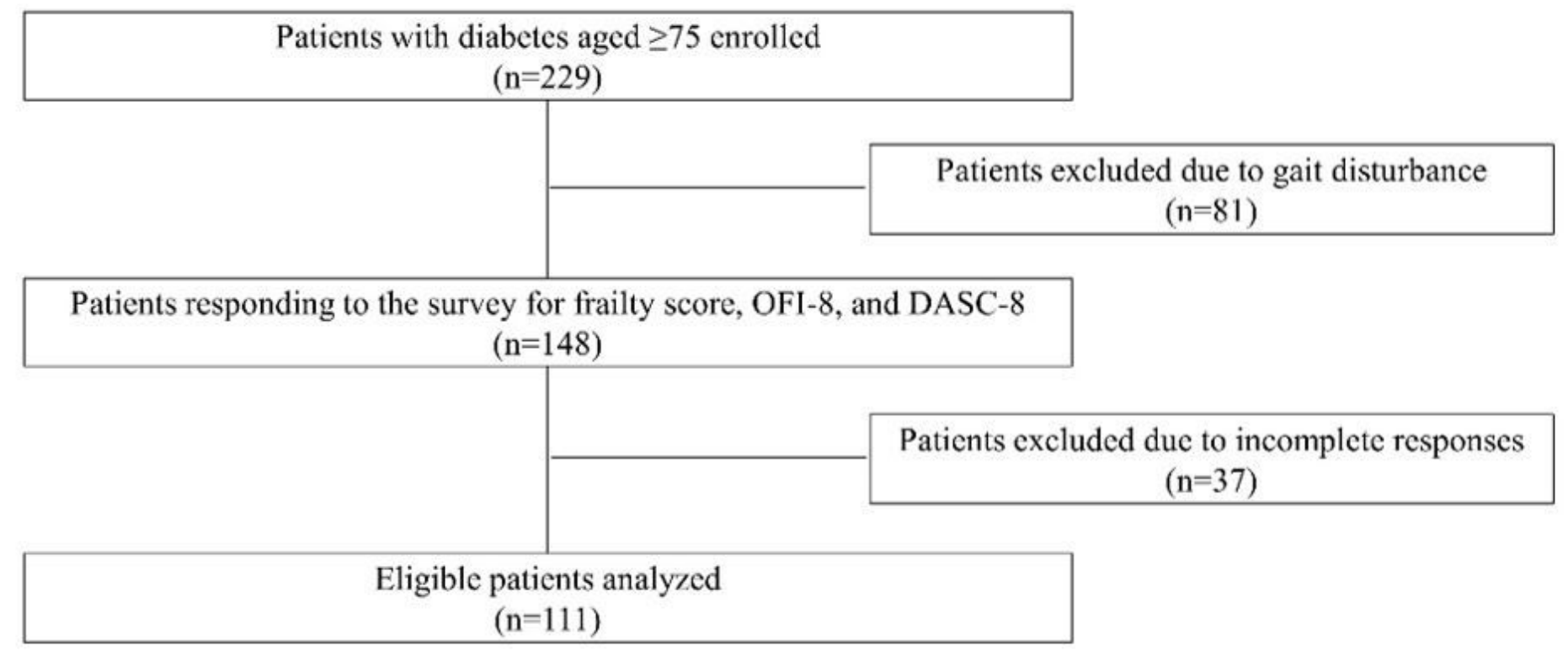

Figure 1

Patient inclusion/exclusion flowchart DASC-8: the dementia assessment sheet for community-based integrated care system-8 items, OFI-8: oral frailty index-8

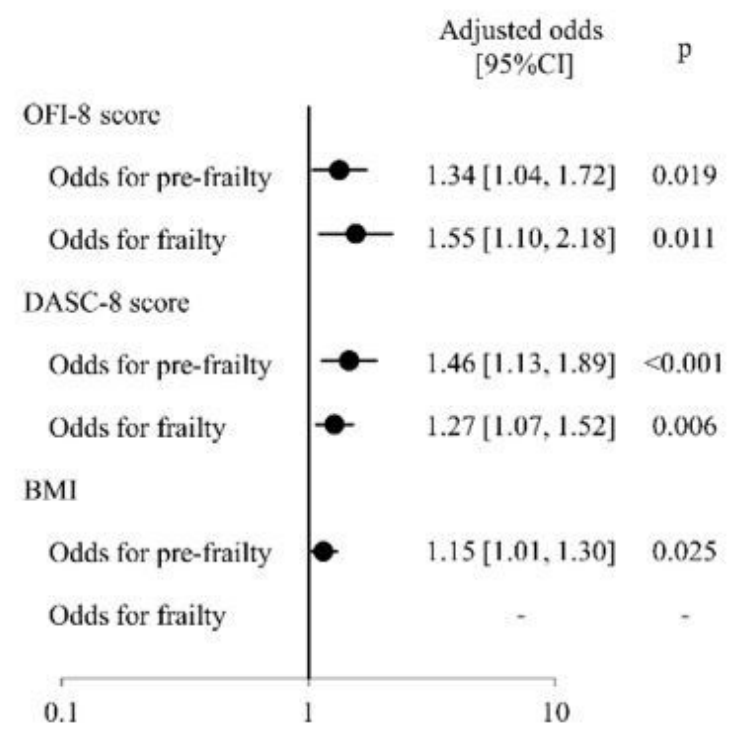

a. Odds ratios for pre-frailty and frailty

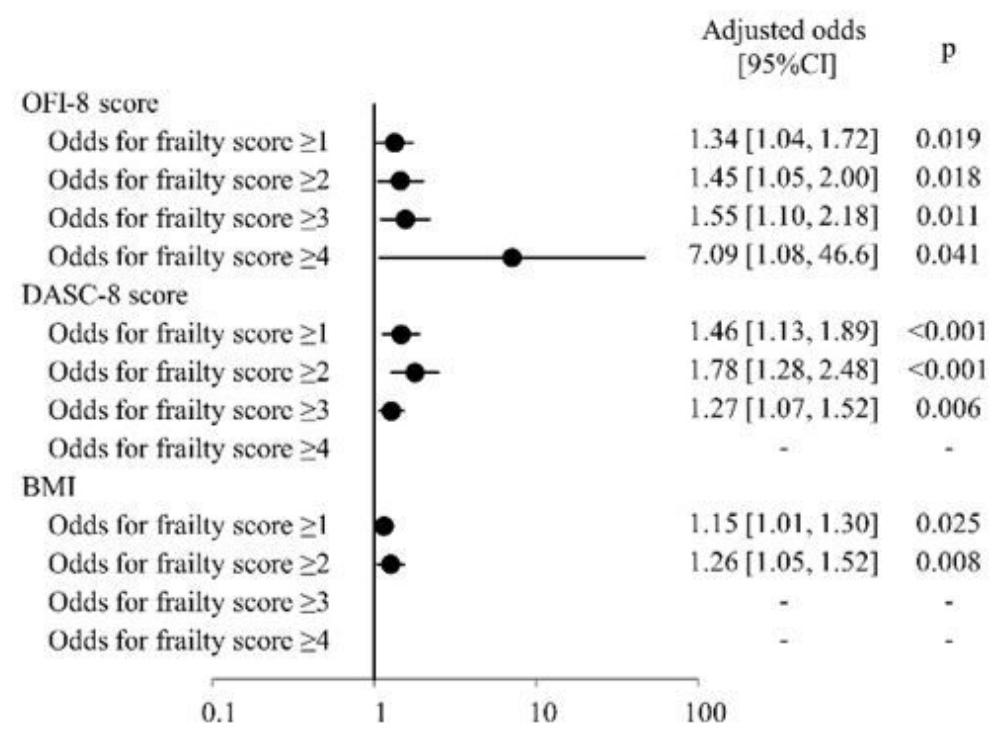

b. Odds ratios by frailty score category

\section{Figure 2}

Adjusted odds ratios for frailty DASC-8: the dementia assessment sheet for community-based integrated care system-8 items, OFI-8: oral frailty index-8 


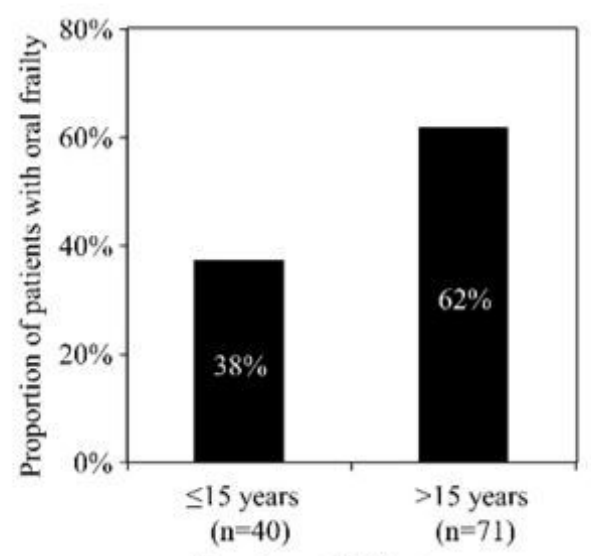

Duration of diabetes

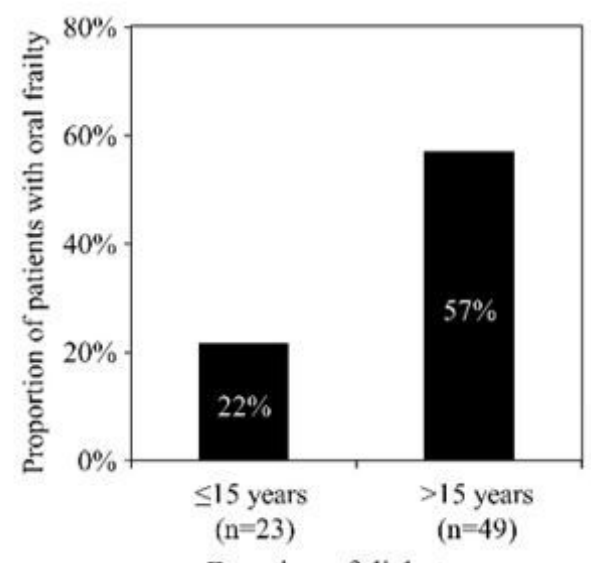

Duration of diabetes

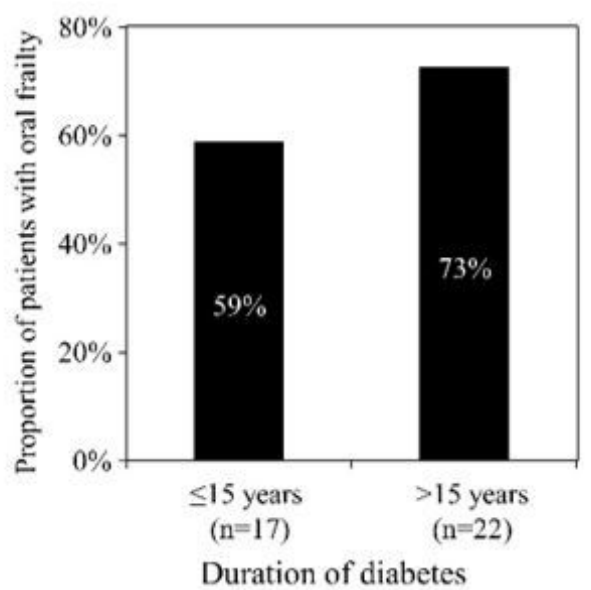

b. Male

c. Female

\section{Figure 3}

Duration of diabetes and proportion of patients with oral frailty Oral frailty was defined as an oral frailty score $\geq 4$ 